\title{
Neural Changes after Operant Conditioning of the Aerial Respiratory Behavior in Lymnaea stagnalis
}

\author{
Gaynor E. Spencer, ${ }^{1}$ Naweed I. Syed, ${ }^{1}$ and Ken Lukowiak ${ }^{1,2}$ \\ ${ }^{1}$ Departments of Anatomy, Physiology, and Biophysics, Health Sciences Centre, Calgary, Alberta, Canada T2N 4N1, and \\ ${ }^{2}$ Arabian Gulf University, Manama, Bahrain
}

In this study, we demonstrate neural changes that occurred during operant conditioning of the aerial respiratory behavior of Lymnaea stagnalis. Aerial respiration in Lymnaea occurs at the water interface and is achieved by opening and closing movements of its respiratory orifice, the pneumostome. This behavior is controlled by a central pattern generator (CPG), the neurons of which, as well as the motoneurons innervating the pneumostome, have previously been identified and their synaptic connections well characterized.

The respiratory behavior was operantly conditioned by applying a mechanical stimulus to the open pneumostome whenever the animal attempted to breathe. This negative reinforcement to the open pneumostome resulted in its immediate closure and a significant reduction in the overall respiratory activity. Electrophysiological recordings from the isolated CNSs after operant conditioning showed that the spontaneous patterned respiratory activity of the CPG neurons was significantly reduced. This included reduced spontaneous activity of the CPG interneuron involved in pneumostome opening (input 3 interneuron) and a reduced frequency of spontaneous tonic activity of the CPG interneuron [right pedal dorsal 1 (RPeD1)]. The ability to trigger the patterned respiratory activity by electrical stimulation of RPeD1 was also significantly reduced after operant conditioning. This study therefore demonstrates significant changes within a CPG that are associated with changes in a rhythmic homeostatic behavior after operant conditioning.

Key words: operant conditioning; learning; invertebrate; aerial respiration; central pattern generator; neuronal mechanisms; interneuron; motoneuron
Operant conditioning is a form of associative learning in which an association is made between an external stimulus and a behavioral response. The operant conditioning paradigm requires an external stimulus to be presented only if and when an animal performs a particular behavior. Such a reinforcing stimulus may be either positive or negative and subsequently alters the associated behavior (see Mackintosh, 1974, for full details of animal learning). Operant conditioning protocols have been used in both vertebrates (Chen and Wolpaw, 1995; Feng-Chen and Wolpaw, 1996) and invertebrates (Horridge, 1962; Hoyle, 1980; Forman, 1984; Hawkins et al., 1985; Susswein et al., 1986; Cook and Carew, 1989a,b,c; Nargeot et al., 1997). In contrast to many classical conditioning studies in which underlying neural mechanisms have been defined (for review, see Carew and Sahley, 1986), little is known of neural correlates of operant conditioning, in part because of the poor definition of certain behaviors and the complexity of the underlying neuronal mechanisms. We have studied aerial respiratory behavior in the fresh water mollusc, Lymnaea stagnalis. Not only is this behavior well defined (Syed and Winlow, 1991; Syed et al., 1991) and easily monitored (Lukowiak et al., 1996), but the underlying neuronal circuit has

Received June 24, 1998; revised Dec. 10, 1998; accepted Dec. 15, 1998.

This work was supported by the Medical Research Council (Canada). N.I.S. is an Alberta Heritage Foundation for Medical Research Senior Scholar. G.E.S. is a Killam, University of Calgary, and an Alberta Heritage Foundation for Medical Research Fellow. We acknowledge E. Ringseis, B. Salkeld, R. Achal, S. Poon, R. Cotter, J. Westly, and A. Oonwala for their contributions toward animal training and W. Zaidi and T. Lee for technical assistance. We also thank Z. Topor for statistical advice and Dr. R. Hawkes for critical review of this manuscript.

Correspondence should be addressed to Dr. G. E. Spencer, Department of Anatomy, Health Sciences Centre, 3330 Hospital Drive N.W., Calgary, Alberta, Canada T2N 4N1.

Copyright (C) 1999 Society for Neuroscience $\quad 0270-6474 / 99 / 191836-08 \$ 05.00 / 0$ also been extensively characterized (Syed et al., 1990, 1991; Syed and Winlow, 1991).

Lymnaea is a bimodal breather and exchanges gases either through its skin or via its lung (aerial respiration). Aerial respiration occurs when the water becomes hypoxic and the animal acquires oxygen at the air-water interface by opening and closing movements of its pulmonary orifice, the pneumostome (Syed et al., 1991). Aerial respiration is controlled by a well defined respiratory central pattern generator (CPG) (Syed and Winlow, 1991; Syed et al., 1991), which consists of at least three neurons, namely right pedal dorsal 1 (RPeD1), input 3 interneuron (IP3), and visceral dorsal 4 (VD4). This three-neuron network is both sufficient and necessary to produce the respiratory rhythm (Syed et al., 1990). The connectivity and firing patterns of the CPG interneurons and the pneumostome opener motoneurons $(\mathrm{I} / \mathrm{J}$ cells) underlying aerial respiration in Lymnaea have been well documented, providing a well defined circuitry from which to record and monitor neuronal activity. Furthermore, the individually identifiable neurons are amenable to electrophysiological analysis.

In this study, we have taken advantage of these features to investigate the neural mechanisms underlying operant conditioning. We have shown previously that Lymnaea is capable of operant learning and that the memory associated with the operant paradigm can persist for up to 1 month (Lukowiak et al., 1996, 1998). Operant conditioning was achieved by first placing the animals in an hypoxic environment to increase their respiratory drive. Animals in the operant group received tactile stimulation of the open pneumostome whenever they attempted to breathe. This stimulation resulted in immediate closure of the pneumostome and a significant reduction in the overall aerial respiratory 
behavior. That is, after the operant training paradigm, the animals attempted to open their pneumostomes significantly less often, resulting in a significant reduction in both the number of breaths and the total breathing time (for further details of training procedures, see Lukowiak et al., 1996; Materials and Methods).

This work has been presented previously in abstract form (Spencer et al., 1996).

\section{MATERIALS AND METHODS}

Training procedures. Animals were trained as described previously by Lukowiak et al. (1996). The animals were placed in hypoxic pond water at the beginning of each training session. During each training period, a tactile stimulus was presented to the lip of the open pneumostome area every time an animal attempted to breathe (operant group). This stimulus caused immediate closure of the pneumostome. The yoked control animals received the same number of stimuli as their operant counterparts. These stimuli were not contingent on pneumostome openings of the yoked animals, but rather corresponded to the openings of the operant animals and were applied to the lip of the closed pneumostome. Both operant and yoked groups received a total of five training sessions (each $30 \mathrm{~min}$ in duration), with two sessions each day for $2.5 \mathrm{~d}$. After the five training sessions, only the operant group showed a significant reduction in the number of breaths (see Fig. 2A). Previous studies found no significant differences in respiratory activity of control animals that were prevented from breathing at the surface of the water for the 30 min test period (hypoxic controls) (Lukowiak et al., 1996).

Dissection procedures. Immediately after the fifth training session, the animals were anesthetized (30\% Listerine) and deshelled, and the CNSs were dissected and pinned dorsal surface uppermost in an enclosed recording chamber containing HEPES buffered and nonoxygenated saline (Inoue et al., 1996a). After proteolytic enzymatic treatment (pronase E; Sigma, Toronto, ON), the outer ganglionic sheath was removed using a pair of fine forceps.

Electrophysiological recordings. Standard electrophysiological techniques were used for simultaneous intracellular recordings from both the respiratory interneuron, RPeD1, and a pneumostome opener motoneuron (I or J cell). Signals were amplified via Dagan (Minneapolis, MN) amplifiers and displayed on a Tektronix (5A14N; Wilsonville, OR)) oscilloscope, and recordings were obtained on a Gould chart recorder. The resting membrane potential (RMP) and firing frequency of RPeD1 were measured for at least $20 \mathrm{~min}$. The activity of the IP3 interneuron was monitored and analyzed indirectly as a well characterized excitatory discharge in both RPeD1 and the I/J opener motoneurons, and the number, duration, and interburst interval of IP3 bursting activity were measured for the same time period. The ability of electrical stimulation of RPeD1 $(1-5 \mathrm{nA}, 5 \mathrm{sec})$ to trigger IP3 activity in both the RPeD1 and $\mathrm{I} / \mathrm{J}$ cells was also tested.

All data were analyzed over a $400 \mathrm{sec}$ period of each recording and were statistically analyzed using Student's $t$ test, or when the normality test failed, the Mann-Whitney rank sum test was used. For data sets that reflected either the absence or presence of a phenomenon, Fisher's exact test was used to determine significance (SigmaStat; Jandel Scientific, San Rafael, CA). All values are expressed as either mean \pm SEM or as a percentage. All electrophysiological recordings and data analyses were performed blind.

\section{RESULTS}

The CPG controlling aerial respiration in Lymnaea consists of three neurons, namely, RPeD1, VD4, and IP3. The motoneurons innervating pneumostome opener and closer muscles have also been identified and characterized. These motoneurons are the I or J (opener) and $\mathrm{K}$ (closer) cells of the visceral ganglion (Benjamin and Winlow, 1981; Syed and Winlow, 1991; Syed et al., 1991). A summary diagram depicting synaptic connections between the respiratory CPG interneurons and motoneurons is shown in Figure 1.

RPeD1 first receives excitatory afferent chemosensory and mechanosensory input from the pneumostome-osphradial area, which, in turn, has been shown to initiate the CPG activity and

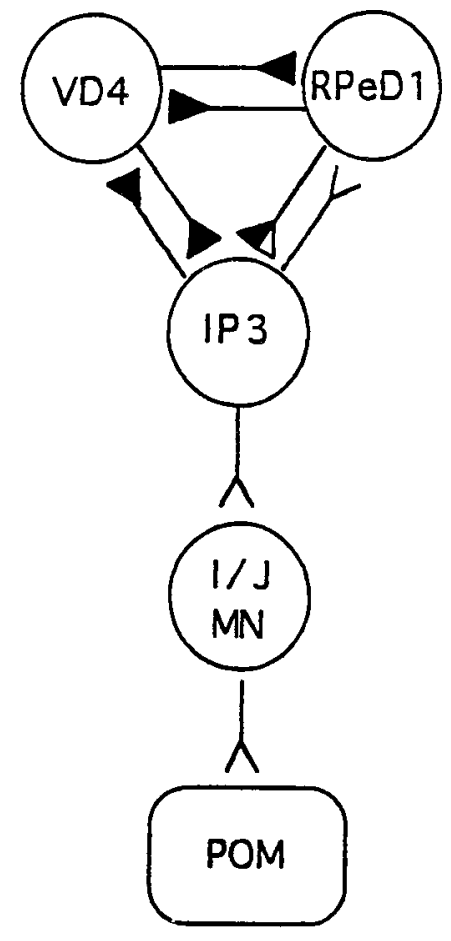

Figure 1. Synaptic connections between the respiratory CPG neurons and pneumostome opener motoneurons. VD4, Visceral dorsal 4; RPeD1, right pedal dorsal $1 ; I P 3$, input $3 ; I / J M N$, I/J motoneuron; $P O M$, pneumostome opener muscles. Excitatory connections are represented as open triangles, whereas inhibitory connections are represented as filled triangles. $\mathrm{RPeD1}$ receives afferent chemosensory and mechanosensory input from the periphery and excites IP3 biphasically (inhibition followed by excitation). The IP3 interneuron controls pneumostome opening and has excitatory connections with both RPeD1 and the I/J pneumostome motoneurons.

hence the respiratory rhythm (Inoue et al., 1996b). IP3 and VD4, which control the opening (expiration) and closing (inspiration) of the pneumostome, respectively (Syed and Winlow, 1991; Park and Winlow, 1993), have reciprocal inhibitory connections with each other. RPeD1 and VD4 also make reciprocal inhibitory connections resembling that of a "half-center" model (Syed and Winlow, 1991). Once stimulated, RPeD1 activates IP3 via a biphasic effect (inhibition followed by excitation). IP3, in turn, excites both RPeD1 (which is the only central excitatory input $\mathrm{RPeD} 1$ is known to receive) and the I/J motoneurons. The IP3 interneuron produces distinctive discharges in the $\mathrm{I} / \mathrm{J}$ motoneurons (Syed et al., 1991); no other input producing such a discharge pattern in these cells has been identified in the CNS. IP3-induced action potentials in the $\mathrm{I} / \mathrm{J}$ motoneurons produce $1: 1$ excitatory junction potentials in the pneumostome muscles and hence produce pneumostome opening (Syed and Winlow, 1991). IP3 also produces an inhibitory effect on VD4, and after release from this inhibition, VD4 fires, resulting in pneumostome closure. In semiintact and isolated brain preparations, the three CPG neurons fire spontaneous, alternating bursts of action potentials, which produce the rhythmical activity underlying the aerial respiratory behavior. This respiratory behavior can be initiated in semi-intact preparations by electrical stimulation of RPeD1, resulting in pneumostome opening. Electrical stimulation of RPeD1 has also been shown to initiate the patterned respiratory activity in VD4 and IP3 cells of previously quiescent preparations (Syed et al., 1990). 
In this study, we monitored the neuronal activity of the CPG interneurons RPeD1 and IP3, and also the I/J motoneurons, in isolated CNS preparations after operant conditioning of the respiratory behavior. The firing activity of RPeD1 and the I/J motoneurons was monitored by direct electrophysiological recordings from these cells. Because the IP3 interneuron is located on the opposite (ventral) surface of the brain, simultaneous recordings from both RPeD1 and IP3 were not possible. The activity of the IP3 interneuron was therefore monitored indirectly by recording its distinctive excitatory discharges produced in the I/J motoneurons and RPeD1 (Syed et al., 1991). Animals were operantly conditioned for five training sessions (see Materials and Methods; Lukowiak et al., 1996). Figure $2 A$ shows the number of pneumostome openings for all operantly conditioned animals $(n=92)$ over the five training sessions. The number of openings in session $5(2.2 \pm 0.1)$ were significantly less $(t$ test; $p<0.001$; $\mathrm{df}=90)$ than those of session $1(5.2 \pm 0.4)$. Figure $2, B$ and $C$, shows examples of learning curves for operantly conditioned animals $(n=1)$, which were designated as either a "good learner" or a "poor learner", respectively. That is, although both animals demonstrated a "learning trend" over the first four sessions, the poor learner performed poorly on the final training session. All operantly conditioned animals $(n=92)$ and corresponding yoked controls, regardless of their behavioral performance, were dissected after the final training session for electrophysiological analysis. In the initial sections, data from all operantly conditioned animals are presented. Only data collected from animals exhibiting a $50 \%$ reduction in their pneumostome openings by the final session (good learners) are presented in the final section.

\section{A higher percentage of RPeD1 cells were silent in operantly conditioned preparations}

RPeD1 has been shown previously to initiate the respiratory CPG activity (Syed et al., 1990; Syed and Winlow, 1991). We first sought to determine whether operant conditioning of respiratory behavior had altered any of its intrinsic membrane properties. We found no significant differences (Mann-Whitney test; $p>0.05$ ) between the RMP in RPeD1 of the operantly conditioned $(-60.0 \pm 1.2 \mathrm{mV} ; n=61)$ and the yoked control $(-60.0 \pm 1.2$ $\mathrm{mV} ; n=65)$ preparations. Furthermore, there was no significant difference (Mann-Whitney test; $p>0.05$ ) between the overall spontaneous firing frequency of RPeD1 in the operantly conditioned $(0.42 \pm 0.05 \mathrm{~Hz} ; n=92)$ and yoked control $(0.32 \pm 0.04$ $\mathrm{Hz} ; n=76)$ preparations. We did, however, find that RPeD1 was quiescent in significantly more (Fisher's exact test; $p<0.04$; df $=$ $1)$ operantly conditioned $(23.9 \%, n=22 / 92)$ than yoked control (10.5\%; $n=8 / 76)$ preparations (Fig. 3).

\section{IP3 activity was reduced in the operantly conditioned group}

The IP3 interneuron (which controls pneumostome opening) generates distinctive excitatory discharges in both RPeD1 and I/J motoneurons (Fig. 3, top trace). The incidence of spontaneous IP3 activity in RPeD1 and I/J motoneurons was monitored as an indicator of respiratory activity in both operant and yoked control preparations. Figure 3 shows a raw data trace of IP3 activity recorded simultaneously from RPeD1 and a $\mathrm{J}$ motoneuron, in a yoked control and an operantly conditioned preparation. Spontaneous IP3 activity was observed significantly less often (Fisher's exact test; $p<0.005$; df $=1$ ) in RPeD1 cells from operantly conditioned compared with yoked control preparations. That is, spontaneous IP3 discharges were found to be absent in $36.6 \%$ $(n=26$ of 71$)$ of operant preparations compared with only $13.3 \%$
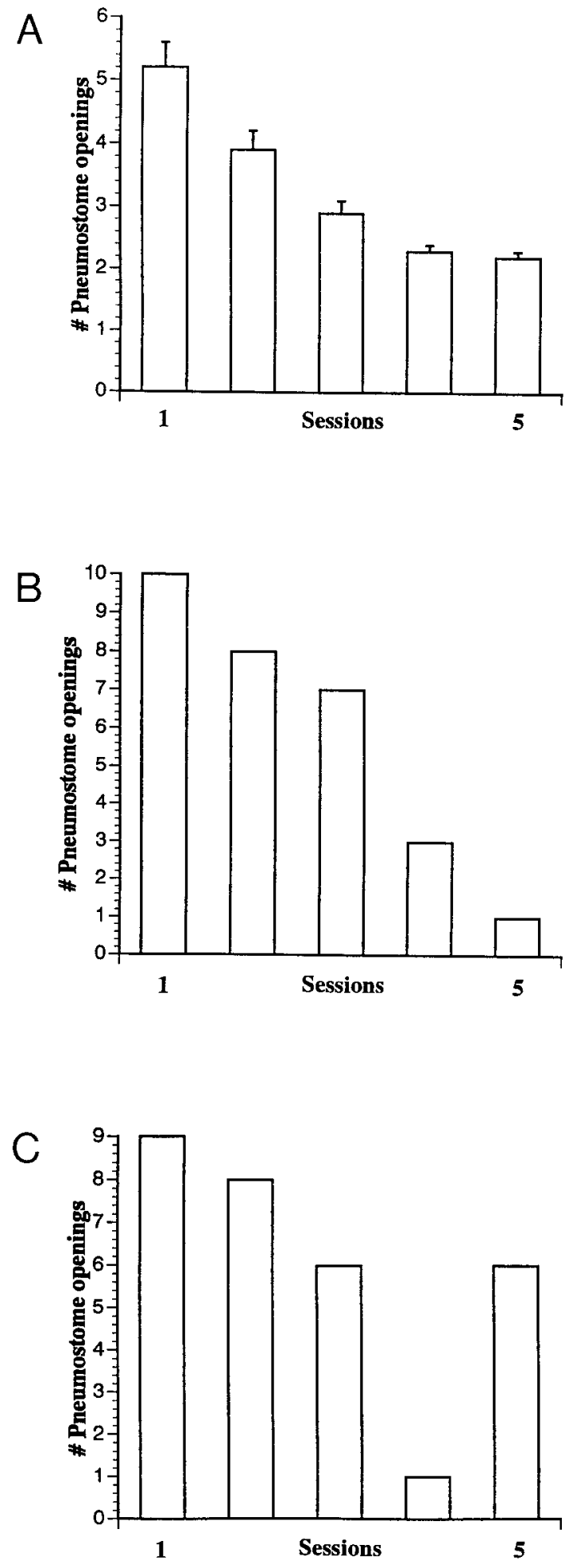

Figure 2. Learning curves showing the number of pneumostome openings over five training sessions. $A$, The mean number of pneumostome openings of all operantly conditioned animals $(n=92)$ was significantly reduced from $5.2 \pm 0.4$ in session 1 to $2.2 \pm 0.1$ in session $5 . B$, A learning curve of an operantly conditioned animal $(n=1)$ representing a good learner. The number of pneumostome openings was reduced from 10 in session 1 to 1 in session 5. $C$, A learning curve of an operantly conditioned animal $(n=1)$ representing a poor learner. The animal showed a learning "trend" over the initial four sessions but performed poorly in the fifth session. The number of pneumostome openings were reduced from nine in session 1 to six in session 5 . 


\section{J cell YOKED CONTROL}
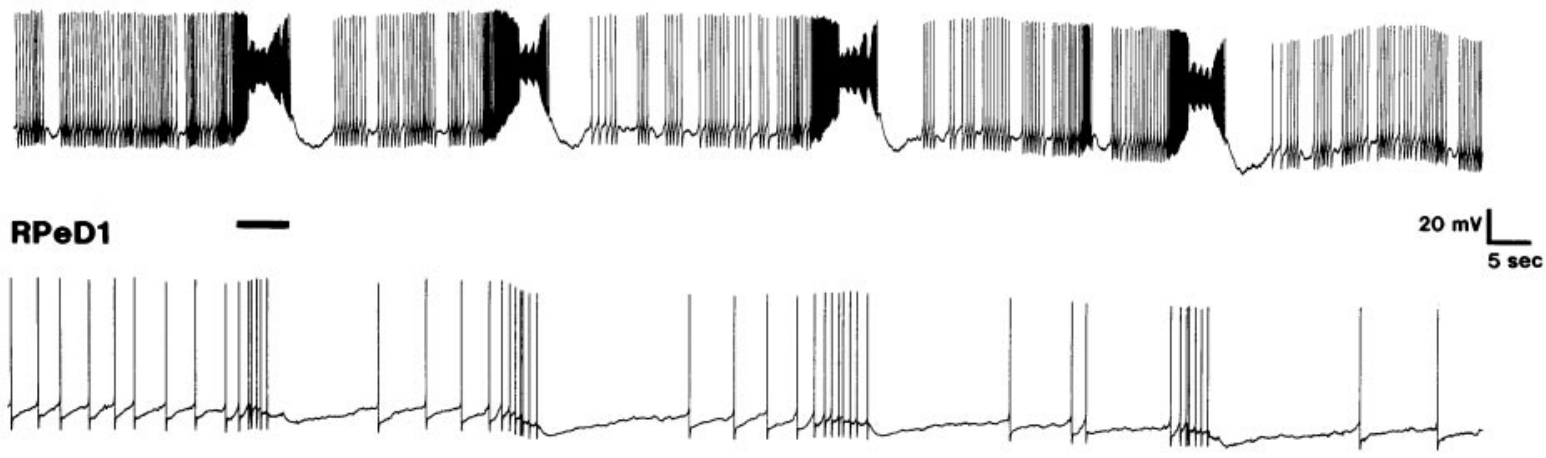

J cell

OPERANTLY CONDITIONED

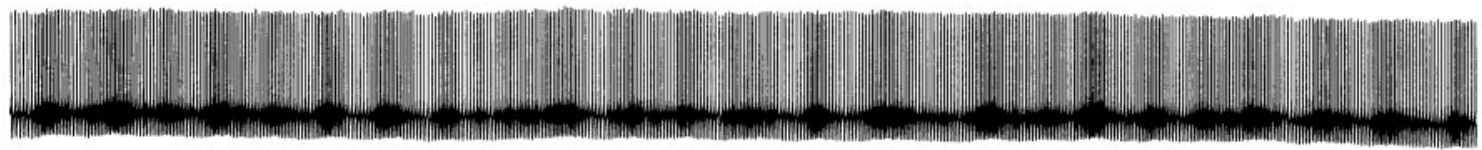

RPeD1

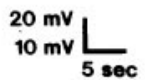

Figure 3. Raw data trace showing simultaneous electrophysiological recordings from a J motoneuron and RPeD1 of both yoked and operant preparations. Note the distinctive rhythmical excitatory IP3 discharges (first burst is marked by a bar) evident in the J cell and the corresponding excitation of RPeD1 in the yoked preparation. The recording from the operant preparation shows the absence of spontaneous IP3 activity in both RPeD1 and $\mathrm{J}$ cells and also the absence of spontaneous firing in RPeD1.

A

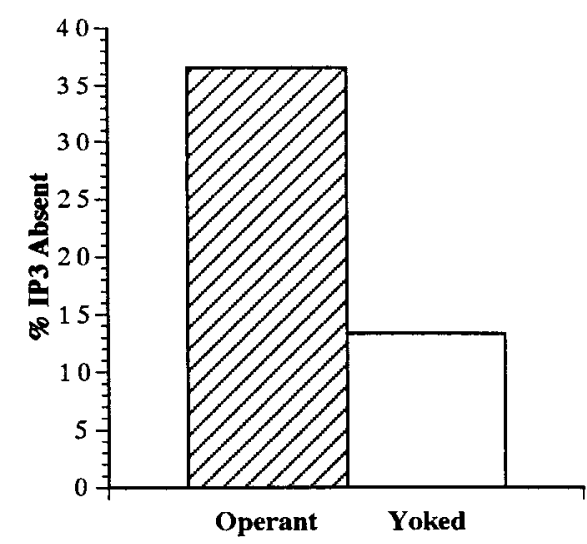

B I/J Cells

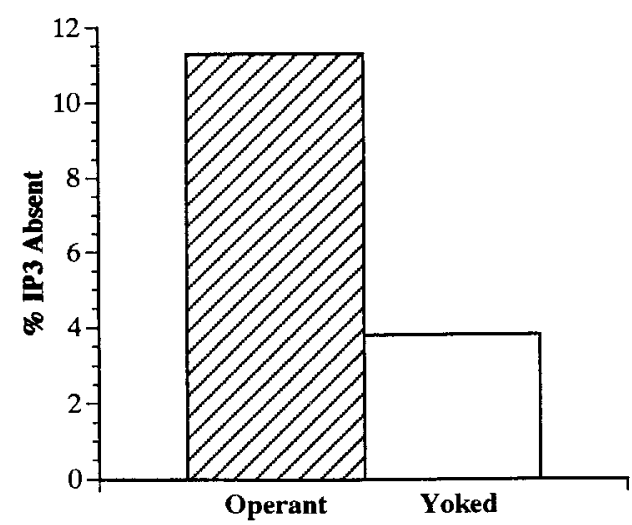

Figure 4. IP3 activity is absent from significantly more operantly conditioned than yoked control preparations. $A$, Bar graph showing the percentage of RPeD1 cells recorded from both operant and yoked preparations in which IP3 activity was absent. In operantly conditioned preparations, a significantly higher $(p<0.005)$ percentage of RPeD1 neurons failed to show IP3 activity. $B$, Bar graph showing the percentage of I/J cells recorded from both operant and yoked preparations in which IP3 activity was absent. A higher percentage of I/J cells from operant preparations failed to show IP3 activity, although this difference was not found to be significant $(p>0.05)$.

$(n=8$ of 60$)$ of yoked control preparations (Fig. $4 A$ ). Furthermore, the corresponding IP3 activity in I/J motoneurons was absent in $11.4 \%$ (9 of 79) of operant preparations compared with only $3.8 \%$ (3 of 77) of yoked control preparations (Fig. $4 B$ ). This difference was not, however, significant (Fisher's exact test; $p>$
0.05; df $=1)$. The number, duration, and interburst intervals of the IP3 inputs in the I/J cells were also measured. We found that the mean number of IP3 bursts recorded from each I/J cell was significantly lower ( $t$ test; $p<0.001$; df $=154)$ in the operant preparations $(15.4 \pm 1.1 ; n=79)$ than in the yoked control 
A

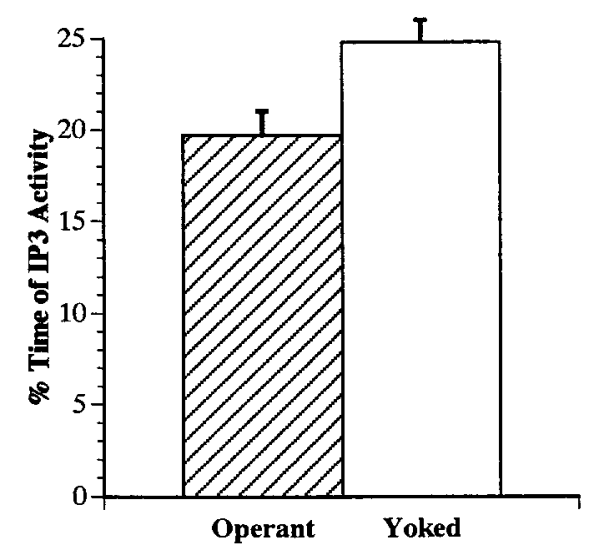

B

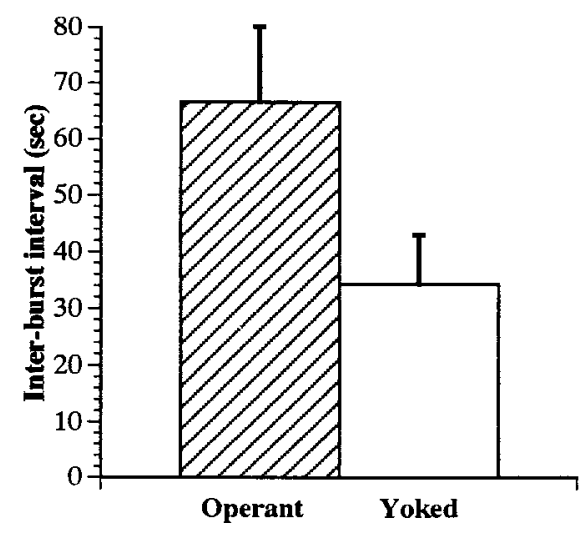

Figure 5. Spontaneously occurring IP3 activity recorded from the I/J motoneurons was reduced in operantly conditioned preparations. $A$, Bar graph showing a significant reduction $(p<0.005)$ in the incidence of IP3 activity (percentage of total time occupied by IP3 discharges) in operant preparations. $B$, Bar graph showing a significant increase $(p<0.001)$ in the interburst interval (in seconds) of IP3 activity recorded from I/J motoneurons of operant preparations.

$(21.2 \pm 1.2 ; n=77)$ preparations. That is, the time during which the IP3 interneuron was active was significantly reduced ( $t$ test; $p<0.005)$ from $24.8 \pm 1.2 \%$ in the yoked control preparations to $19.7 \pm 1.3 \%$ in the operant preparations (Fig. $5 A$ ). Similarly, the interburst interval was significantly longer (Mann-Whitney test; $p<0.001)$ in the operantly conditioned preparations $(66.6 \pm 13.5$ $\mathrm{sec})$ than in the yoked control preparations $(34.3 \pm 8.7 \mathrm{sec})$ (Fig. $5 B)$. There was no significant difference (Mann-Whitney test; $p>$ $0.05)$ in the duration of the IP3 bursts between operant $(4.5 \pm 0.3$ $\mathrm{sec})$ and yoked control $(4.8 \pm 0.3 \mathrm{sec})$ preparations (data not shown).

\section{RPeD1-induced IP3 activity was reduced in operant preparations}

Electrical stimulation of RPeD1 triggers IP3 activity (and hence the respiratory rhythm) in both an isolated CNS (Syed and Winlow, 1991) and in cell culture (Syed et al., 1990). That is, bursts of spikes in RPeD1 induce a biphasic response (inhibition followed by excitation) in the IP3 interneuron, which in turn excites both RPeD1 and the $I / J$ motoneurons to initiate the respiratory rhythm. Figure 6 shows a raw data trace of the excitatory effects of IP3 on RPeD1 and a J cell after RPeD1 stimulation in a yoked preparation, and the absence of IP3 activity in an operant preparation. Electrical stimulation of RPeD1 (1-5 nA, $5 \mathrm{sec})$ produced IP3 activity in $84.2 \%(n=48$ of $57)$ of RPeD1 cells in yoked preparations but in only $38.6 \%(n=$ 22 of 57) of RPeD1 cells in operant preparations (Fisher's exact test; $p<0.001 ; \mathrm{df}=1$ ) (Fig. $7 A$ ). Likewise, stimulation of RPeD1 produced corresponding IP3 activity in $89.5 \%(n=51$ of 57$)$ of $\mathrm{I} / \mathrm{J}$ motoneurons of yoked preparations but in only $52.6 \%(n=30$ of 57) of I/J motoneurons of operant preparations (Fisher's exact test; $p<0.001$; df $=1$ ) (Fig. 7B). Because previous studies have demonstrated that connections between RPeD1 and IP3 interneurons are direct and monosynaptic, these data suggest that the efficacy of synaptic transmission between RPeD1 and IP3 may be altered as a consequence of the operant conditioning paradigm.

\section{IP3 duration was significantly reduced in animals, showing a $\mathbf{5 0} \%$ reduction in respiratory behavior over the five-session training period}

In the previous sections, all animals trained using the operant procedure were analyzed for changes in neuronal activity, and no

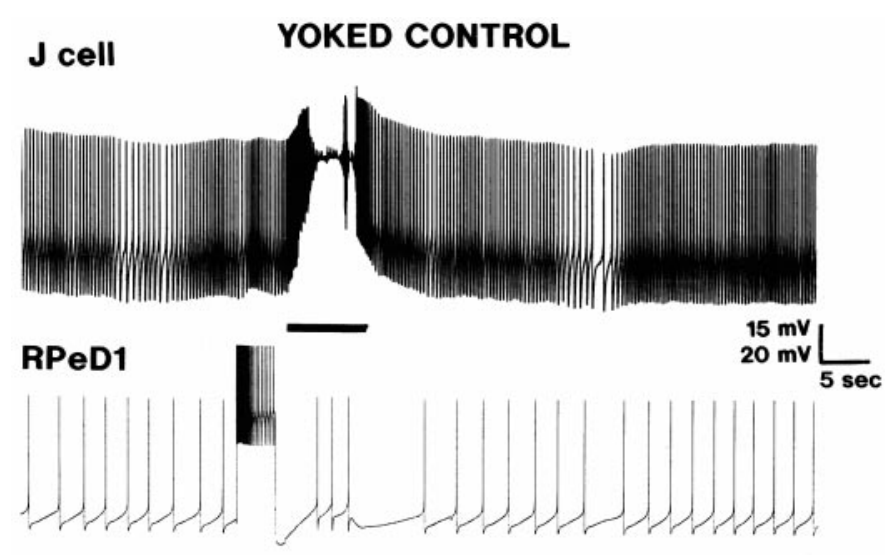

\section{J cell OPERANTLY CONDITIONED}

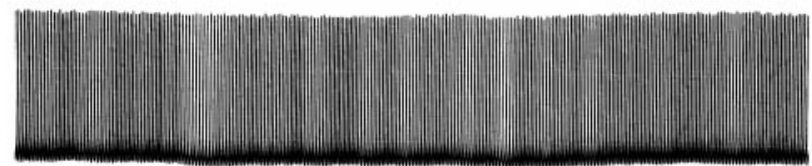

\section{RPeD1}
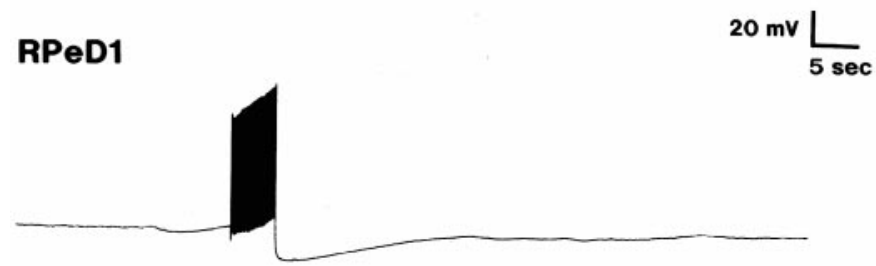

Figure 6. Raw data trace showing the absence of RPeD1-induced IP3 activity in the operantly conditioned preparation. Raw data trace showing that electrical stimulation of RPeD1 (1-5 nA, $5 \mathrm{sec})$ induced IP3 activity (marked by bar) in both the $\mathrm{J}$ motoneuron and RPeD1 from a yoked control preparation. Stimulation of RPeD1 failed to induce IP3 activity in both the $\mathrm{J}$ and RPeD1 cells in an operant preparation. 
A

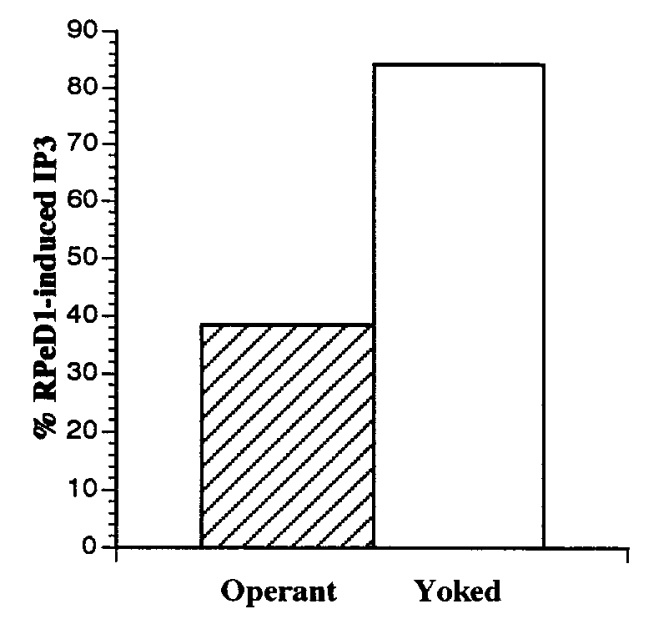

B

I/J Cells

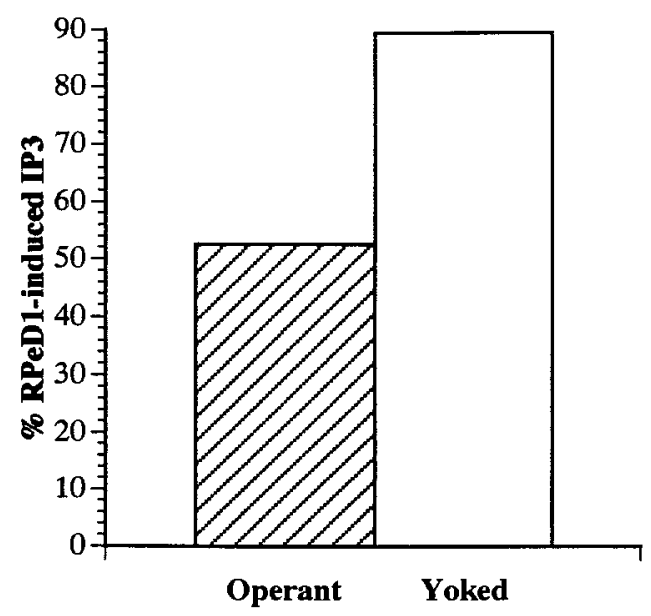

Figure 7. RPeD1 failed to induce IP3 activity in a significantly higher percentage of operantly conditioned than yoked control preparations. $A$, Bar graph showing that electrical stimulation of RPeD1 induced IP3 activity in only $38.6 \%$ of operant RPeD1s compared with $84.2 \%$ of yoked RPeD1s. $B$, Bar graph showing that electrical stimulation of RPeD1 induced IP3 activity in $52.6 \%$ of operant I/J cells compared with $89.5 \%$ of yoked I/J cells.

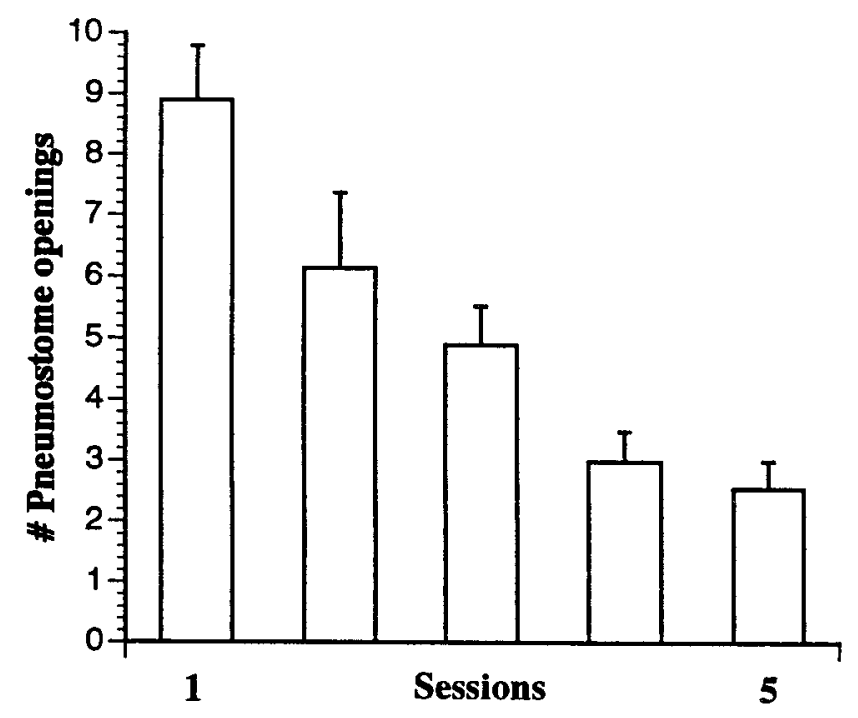

Figure 8. A learning curve representing the best-trained animals. The number of pneumostome openings of the operantly conditioned animals $(n=20)$ was significantly reduced $(p<0.0001)$ from $8.9 \pm 0.9$ in training session 1 to $2.6 \pm 0.5$ in training session 5 .

learning criteria were adopted before analysis of the CPG activity. In the following series of experiments, we analyzed data only from a subset of these animals that expressed a significant reduction in their respiratory activity after the final training session. Only animals that showed $>50 \%$ reduction in their aerial respiratory behavior by the final training session were used (Fig. $2 B$, learning curve). The number of breaths taken in session $5(2.6 \pm$ $0.5 ; n=20$ ) were found to be significantly lower ( $t$ test; $p<$ $0.0001 ; \mathrm{df}=38)$ than those taken in session $1(8.9 \pm 0.9 ; n=20)$. These behavioral data are shown in Figure 8, learning curve.

Differences in IP3 activity were found between operant and yoked preparations, as in the previous sections. The percentage of time during which IP3 interneuron was active (as measured from the $\mathrm{I} / \mathrm{J}$ motoneurons) was significantly reduced ( $t$ test; $p<0.009$; $\mathrm{df}=38$ ) in operant preparations (operant, $16.8 \pm 2.6 \%$; yoked,
$25.6 \pm 1.8 \% ; n=20$ ) (Fig. $9 A$ ). Furthermore, spontaneous IP3 activity was absent from $25 \%$ of operant preparations $(n=5$ of $20)$ compared with $0 \%$ of the yoked controls $(n=0$ of 20 ; Fisher's exact test; $p<0.04$; df $=1$ ). A new finding using these "well trained" animals was that the duration of the IP3 bursts recorded from the $\mathrm{I} / \mathrm{J}$ cells was also significantly reduced ( $t$ test; $p<0.001$; $\mathrm{df}=38)$, from $5.5 \pm 0.5 \mathrm{sec}(n=20)$ in yoked preparations to $3.1 \pm 0.5 \mathrm{sec}(n=20)$ in operantly conditioned preparations (Fig. $9 B)$. No significant changes were found in either the RMP of RPeD1 (operant, $-57.3 \pm 1.8 \mathrm{mV} ; n=20$; yoked, $-55.4 \pm 1.5$ $\mathrm{mV} ; n=20 ; t$ test; $p>0.05$; df $=38$ ) or its firing frequency (operant, $0.37 \pm 0.09 \mathrm{~Hz} ; n=20$; yoked, $0.43 \pm 0.10 \mathrm{~Hz} ; n=20$; Mann-Whitney test; $p>0.05$ ). However, despite no statistically significant change in the overall firing frequency, $25 \%$ of RPeD1 cells $(n=5$ of 20 ) in the operant group were found to be silent compared with $0 \%$ of yoked controls $(n=0$ of 20 ; Fisher's exact test; $p<0.04$; df = 1).

\section{DISCUSSION}

Lymnaea stagnalis was previously shown to be capable of associative learning after classical conditioning of the feeding behavior (Kemenes and Benjamin, 1994; Kojima et al., 1997). Aerial respiration, however, is the first behavior to be operantly conditioned in this animal (Lukowiak et al., 1996). In this study, we have demonstrated significant changes in the properties of a CPG network that corresponded to the reduced respiratory activity that was observed in the operantly conditioned animal.

Little is presently known about neural correlates of operant conditioning in most animals. Feng-Chen and Wolpaw (1996) recently demonstrated that operant conditioning of the primate triceps H-reflex involved local changes within the spinal cord. In Aplysia, Cook and Carew (1989c) identified possible central pathways involved in the operant conditioning of the head-waving response, but no direct neural correlation was made. The first direct neural correlation of an operantly conditioned behavior was demonstrated for Horridge's (1962) shock-avoidance legpositioning paradigm in insects. Because these studies were conducted in headless preparations, it was assumed that the learning occurred elsewhere in the nervous system. It was later shown that 
A

Figure 9. The incidence of IP3 (percentage of total time), as well as its burst duration, were significantly reduced in operant animals, showing a significant reduction in the overall respiratory activity. $A$, Bar graph showing a significant $(p<0.009)$ reduction in the incidence of IP3 discharge (percentage of total time occupied by IP3 discharges) in operant preparations $(n=20)$. B, Bar graph showing a significant $(p<0.001)$ reduction in the duration of IP3 bursts in the $\mathrm{I} / \mathrm{J}$ motoneurons of operant preparations $(n=20)$.

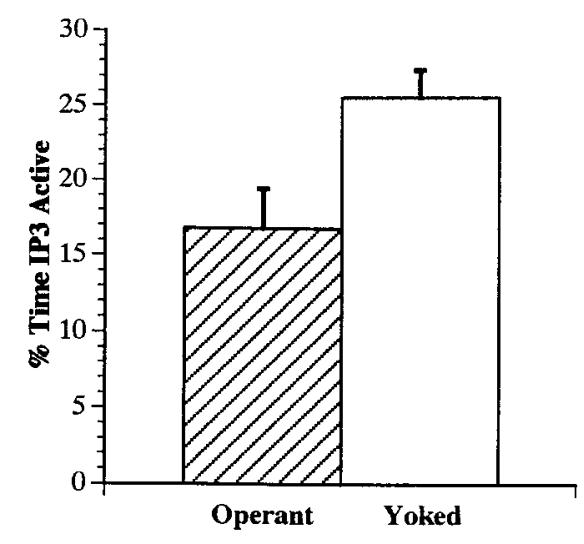

B

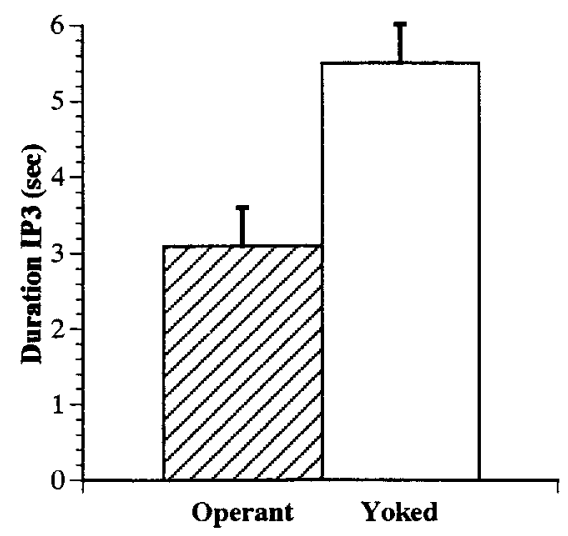

conditioning of leg positioning alters the pacemaker rhythm of a metathoracic ganglion motoneuron innervating the specific leg muscles (Woollacott and Hoyle, 1977). More recent progress in understanding cellular mechanisms of operant conditioning have used the defensive reflex of Helix, and these studies demonstrated that conditioning of the discharge of a single neuron could determine the action of the entire preparation (Tsitolovsky and Shvedov, 1997). Nargeot et al. (1997) also used the isolated buccal preparation of Aplysia to develop an in vitro analog of operant conditioning to demonstrate changes in the activity of the feeding CPG.

We have shown previously that the aerial respiratory behavior in Lymnaea can be operantly conditioned (Lukowiak et al., 1996). Specifically, we demonstrated that only the operantly conditioned group reduced their number of breaths and total breathing time over a period of five training sessions. In contrast to experimental animals, the yoked controls received tactile stimuli to their pneumostomes in a closed state. It is, therefore, plausible that the tactile stimulation of an open pneumostome may have proven more aversive, causing a nonassociative suppression of the behavior (Lukowiak et al., 1996).

In this study, we investigated changes in the neuronal properties of the CPG neurons controlling aerial respiration in isolated CNS preparations. Because RPeD1 receives input from the periphery and, in turn, triggers the respiratory rhythm, it was first hypothesized that a change in the membrane properties of RPeD1 may underlie the behavioral changes associated with learning. The electrophysiological data presented in this study revealed no significant changes in either the RMP or overall firing frequency of RPeD1 in the operantly conditioned preparations. However, a significantly higher percentage of RPeD1 cells from operant preparations was found to be quiescent. This percentage of RPeD1 cells lacking spontaneous activity was increased when the "best-trained" animals were analyzed, although this did not exceed $25 \%$ of the population. The behavioral data, on the other hand, indicated a reduction but not a complete absence of respiratory activity. The reduced activity in RPeD1 may also have led to a reduction in excitability of other neurons within the network, possibly altering the overall output of the CPG-motoneuron network. Whether learning and memory can result from changes in the excitability of just one neuron or involves multiple neurons within a network remains unclear. Interestingly, previous studies have shown that learning procedures directed at one particular neuron can influence other presynaptic and postsynaptic neurons underlying that same behavior (Tsitolovsky and Shvedov, 1997).
In Lymnaea, rhythmical discharges in IP3 are required for the respiratory behavior. In this study, we demonstrated that, in addition to a reduction in RPeD1 activity, the number of spontaneous IP3 discharges (measured from both the RPeD1 neuron and the I/J motoneurons) was also significantly reduced. That is, the time during which IP3 was active, the number of bursts, and the interburst intervals were all significantly changed in the operant preparations. These changes corresponded well with the behavioral observations made from operantly conditioned animals. Furthermore, when the best-trained animals were analyzed, operant conditioning also induced a reduction in the mean duration of the IP3 bursts. The fact that only the best-trained animals exhibited this particular change in their neural properties suggests that this parameter may prove to be an important neural correlate for learning.

Syed and Winlow (1991) have shown that animals kept in an oxygen-deprived environment exhibit more spontaneous respiratory patterned activity within the CPG than well aerated animals. However, after the operant training during which the animals were kept in hypoxic pond water, the level of spontaneous firing of the IP3 interneuron was reduced. The observed changes in the IP3 activity do not appear to be associated with a hypoxic response (Lukowiak et al., 1996), although this possibility remains to be ruled out entirely. Although the underlying mechanisms are presently unknown, the overall reduction in IP3 activity corresponds to a reduced respiratory activity observed in the intact animal. In semi-intact preparations, mechanical stimulation of the pneumostome during opening has been shown not only to cause its closure but also to inhibit IP3 activity in the middle of its discharge (Syed and Winlow, 1991). It is probable that repeated stimulation of an open pneumostome during the training paradigm lowered the levels of IP3 excitability and activity over the five training sessions. However, because IP3 activity was measured indirectly from the RPeD1 and I/J cells, it is difficult to demonstrate conclusively whether an observed change in IP3 activity resulted from reduced excitability of the IP3 interneuron or from modulation of synaptic efficacy between the cells.

Changes in existing synaptic connections are well documented for classical conditioning and nonassociative learning in both vertebrates and invertebrates (for review, see Greenough and Bailey, 1988), although similar observations after operant training paradigms are scarce (for example, see Feng-Chen and Wolpaw, 1996), and none have previously been demonstrated in an invertebrate preparation. In our study, a significant reduction in RPeD1-induced IP3 activity was observed in operant prepara- 
tions. However, whether this neural change actually underlies learning remains to be determined. This reduction in RPeD1induced IP3 activity may have resulted from modulation of synaptic efficacy between RPeD1 and IP3 or from modulation of the synaptic connections between IP3 and its postsynaptic RPeD1, I, and $\mathrm{J}$ cells. Although the neuronal changes in this study (RPeD1 and IP3 interneurons) may be sufficient to explain the observed behavioral changes in the intact animal, we cannot rule out the possibility that discrete changes in other neurons within the CPG network may also have occurred. This notion is consistent with the earlier studies of Lockery and Sejnowski (1993), who proposed that memory for nonassociative learning may be encoded by small changes dispersed across an entire network. Furthermore, the fact that only $25 \%$ of operant preparations displayed certain modified properties of their CPG neurons (for example, a quiescent RPeD1) suggests that individual animals may use different neuronal pathways to modulate any given behavioral repertoire.

In Lymnaea, a higher order whole-body withdrawal interneuron, right pedal dorsal 11 (RPeD11), has been shown to inhibit the respiratory pattern-generating neurons (Inoue et al., 1996a), and a putative mechanosensory neuron that innervates RPeD11 has also been characterized (Inoue et al., 1996c). It is entirely possible that these neurons, as well as others not yet identified, may act to inhibit the activity of the CPG neurons (RPeD1 or IP3 neurons). Because the whole-body withdrawal circuit is well characterized and its synaptic connections with the respiratory circuit are defined (Inoue et al., 1996a), the potential role of such a circuit in the conditioned behavior can now be determined.

In conclusion, we have demonstrated significant changes in the properties of a CPG network that correspond to the reduced respiratory activity observed in the operantly conditioned animal. These studies demonstrate, however, that the neuronal changes appear to be dispersed across the entire network. Future challenges will therefore be to identify the precise loci at which these changes occur and to also determine whether and/or how such changes correlate to the acquisition and/or maintenance of the learned behavior.

\section{REFERENCES}

Benjamin PR, Winlow W (1981) The distribution of three wide-acting synaptic inputs to identified neurons in the isolated brain of Lymnaea stagnalis (L.). Comp Biochem Physiol 70A:293-307.

Carew TJ, Sahley CL (1986) Invertebrate learning and memory: from behavior to molecules. Annu Rev Neurosci 9:435-487.

Chen KY, Wolpaw JR (1995) Operant conditioning of H-reflex in freely moving rats. J Neurophysiol 73:411-415.

Cook DG, Carew TJ (1989a) Operant conditioning of head-waving in Aplysia. I. Identified muscles involved in the operant response. J Neurosci 9:3097-3106.

Cook DG, Carew TJ (1989b) Operant conditioning of head-waving in Aplysia. II. Contingent modification of electromyographic activity in identified muscles. J Neurosci 9:3107-3114.

Cook DG, Carew TJ (1989c) Operant conditioning of head-waving in Aplysia. III. Cellular analysis of possible reinforcement pathways. J Neurosci 9:3115-3122.

Feng-Chen KC, Wolpaw JR (1996) Operant conditioning of H-reflex changes synaptic terminals on primate motoneurons. Proc Natl Acad Sci USA 93:9206-9211.
Forman RR (1984) Leg position learning by an insect. I. A heat avoidance learning paradigm. J Neurobiol 15:127-140.

Greenough WT, Bailey CH (1988) The anatomy of a memory: convergence of results across a diversity of tests. Trends Neurosci 11:142-147.

Hawkins RD, Clark G, Kandel E (1985) Operant conditioning and differential classical conditioning of gill withdrawal in Aplysia. Soc Neurosci Abstr 11:796.

Horridge GA (1962) Learning of leg position by headless insects. Nature 193:697-698.

Hoyle G (1980) Learning, using natural reinforcements, in insect preparations that permit cellular neuronal analysis. J Neurobiol 11:323-354

Inoue T, Takasaki M, Lukowiak K, Syed NI (1996a) Inhibition of the respiratory pattern-generating neurons by an identified whole-body withdrawal interneuron of Lymnaea stagnalis. J Exp Biol 199: $1887-1898$.

Inoue T, Haque Z, Takasaki M, Lukowiak K, Syed NI (1996b) Hypoxia induced respiratory patterned activity in Lymnaea originates at the periphery: role of the dopamine cell. Soc Neurosci Abstr 22:1142.

Inoue T, Takasaki M, Lukowiak K, Syed NI (1996c) Identification of a putative mechanosensory neuron in Lymnaea: characterization of its synaptic and functional connections with the whole-body withdrawal interneuron. J Neurophysiol 76: 3230-3238.

Kemenes G, Benjamin PR (1994) Training in a novel environment improves the appetitive learning performance of the snail, Lymnaea stagnalis. Behav Neural Biol 61:139-149.

Kojima S, Nanakamura H, Nagayama S, Fujito Y, Ito E (1997) Enhancement of an inhibitory input to the feeding central pattern generator in Lymnaea stagnalis during conditioned taste-aversion learning. Neurosci Lett 230:179-182.

Lockery SR, Sejnowski TJ (1993) A lower bound on the detectability of nonassociative learning in the local bending reflex of the medicinal leech. Behav Neural Biol 59:208-224.

Lukowiak K, Cotter R, Westly J, Ringseis E, Spencer G, Syed NI (1998) Long term memory of an operantly conditioned respiratory behaviour in Lymnaea stagnalis. J Exp Biol 201:877-882.

Lukowiak K, Ringseis E, Spencer G, Wildering W, Syed N (1996) Operant conditioning of aerial respiratory behaviour in Lymnaea stagnalis. J Exp Biol 199:683-691.

Mackintosh NJ (1974) The psychology of animal learning. London: Academic.

Nargeot R, Baxter DA, Byrne JH (1997) Contingent-dependent enhancement of rhythmic motor patterns: an in vitro analog of operant conditioning. J Neurosci 17:8093-8105.

Park J-H, Winlow W (1993) Central and peripheral control of pneumostome movements in Lymnaea. J Physiol (Lond) 467:138P.

Spencer GE, Inoue T, Cotter R, Syed NI, Lukowiak K (1996) Alterations in synaptic activity to and within a CPG may underlie operant conditioning of aerial respiratory behaviour in Lymnaea. Soc Neurosci Abstr 22:1404.

Susswein AJ, Schwartz M, Feldman E (1986) Learned changes of feeding behavior in Aplysia in response to edible and inedible foods. J Neurosci 6:1513-1527.

Syed NI, Winlow W (1991) Respiratory behavior in the pond snail Lymnaea stagnalis II. Neural elements of the central pattern generator (CPG). J Comp Physiol [A] 169:557-568.

Syed NI, Bulloch AGM, Lukowiak K (1990) In vitro reconstruction of the respiratory central pattern generator of the mollusk Lymnaea. Science 250:282-285.

Syed NI, Harrison D, Winlow W (1991) Respiratory behavior in the pond snail Lymnaea stagnalis. I. Behavioral analysis and the identification of motor neurons. J Comp Physiol [A] 169:541-555.

Tsitolovsky LE, Shvedov A (1997) Instrumental conditioning of the activity of putative command neurons in the mollusk Helix. Brain Res 745:271-282.

Woollacott M, Hoyle G (1977) Neural events underlying learning in insects: changes in pacemaker. Proc R Soc Lond B Biol Sci 195: $395-415$. 